\title{
A Video Delivery in Wireless Sensor Networks
}

\author{
S. Guo and T.D.C. Little \\ Department of Electrical and Computer Engineering \\ Boston University, Boston, MA 02215 \\ \{guosong,tdcl\}@bu.edu
}

MCL Technical Report No. 01-29-2010

\begin{abstract}
Recent advances in wireless communications technology and low-power, low-cost CMOS imaging sensors stimulate research on the analysis and design of ubiquitous video sensing and delivery in wireless sensor networks. However, scalable deployments remain limited or impractical. Critical challenges such as radio interference, limited channel capacity, and constrained energy resources are still barriers to large-scale deployment of these wireless video sensor networks. The solution space can be explored in several dimensions including data compression, video image analysis and extraction, and intelligent data routing. In this chapter we focus on the analysis of video delivery and data routing techniques for wireless video sensor networks. Our work is intended to inspire additional efforts leading to video routing techniques optimized to different topologies, the physical medium, network channels, and energy constraints.
\end{abstract}

Keywords - Wireless Sensor Network (WSN), Wireless Video Sensor Network (WVSN), QoS, Video Streaming, Video Routing.

In Streaming Media Architectures, Techniques, and Applications: Recent Advances, IGI Global (book chapter), 2010. This material is based upon work support by the National Science Foundation under Grant No. CNS-0435353. 


\section{Introduction}

Advances in computer and network technology have led to wireless sensor networks - networks comprised of many small, low-power embedded processors capable of sensing and communicating using short-range networking. Today, sensor networking has emerged as a frontier interconnecting the Internet to the physical world. For example, one can deploy a series of moisture sensors to monitor soil moisture on a farm, for water management, or in smart grid applications to monitor and control lighting in a home or business. Among the many sensor modalities supported by the sensor devices (or motessensor nodes), we concentrate our focus on ones that produce single or multiple images in a video stream. With the development of low-power, low-cost CMOS imaging sensors, scientists envision great potential for multimedia streaming applications of wireless sensor networks in the areas of homeland security, habitat monitoring, and image-based monitoring and control.

For these applications a sensor node can capture images, audio and/or video information, and send them in a compressed form to a consumer elsewhere on the network. A user need not wait for the download of the entire video sequence but instead can playback the content immediately once data begin to arrive at the receiver. The flexibility of wireless sensor networks coupled with this sensing modality makes video observation very promising to enable humans to observe phenomena or locations that are otherwise difficult or dangerous to access. For instance, ornithologists might deploy such a system in a bird habitat and watch bird behavior without any human disturbance. This kind of network also exhibits value in the areas of military detection and security surveillance. Broadly speaking, the technology is a variant of sensor networking called wireless video sensor networking (WVSN).

Challenges in providing WVSN have to do with resource limitations. The data intensity of video creates several problems: (1) capturing and compressing continuous video is expensive in terms of energy costs at a sensor node, (2) data transmission over multiple hops from a video node to an arbitrarily-located consumer uses a communication channel that is prone to contention, (3) nodes comprising intermediate hops have limited data buffering capacity, and (4) the existence of multiple video sources and video consumers creates resource management complexity.

Much research has been conducted in the field of sensor networking; considerable effort has also been applied to delivering video in networks. Examples include monitoring near-shore environments (Holman et al. 2003), assisted living for elders (Teixeira et al. 2006), deploying large scale surveillance video sensor networks (Chu et al. 2004), people counting and indoor localization (Teixeira et al. 2007), multi-target tracking (Kulkarni et al. 2005) and other uses of vision sensing (Rowe et al. 2007).

Many of the WVSN applications above are an integration of Internet video streaming solutions to the domain of wireless sensor networks. Some applications rely on conventional wired video cameras. Others assume wireless communications but do not address scale-up to large numbers of video cameras nor the support of many streams. Two distinguished applications among the above are SensEye (Kulkarni et al. 2005) and FireFly (Rowe et al. 2007). SensEye uses a multi-tier video solution for pervasive video sensing. The low tier network cooperates with the higher tier network to perform the video sensing task. Experiments demonstrate that this network decomposition can result in energyefficient field sensing. However, the requirement of inter-tier communication and cooperation introduces heterogeneity problems to the network and also increases the complexity and cost of the hardware. FireFly presents an image processing framework with operating system, network and image processing primitives that assist in the development of distributed vision sensing tasks. The success for this application is attributed to the utilization of collision-free TDMA link layer for wireless video 
streaming. However, the required network-wide synchronization scheme for TDMA link restricts the scalability of the network deployment.

The main obstacle for the scalability here is the lack of mechanisms to manage contention among multiple source-to-destination video streams in the context of sensor network energy and communication constraints. The remainder of the chapter surveys the state-of-the-art of video routing schemes applicable to a WVSN with the intent of guiding the development of new video routing protocols for WVSNs.

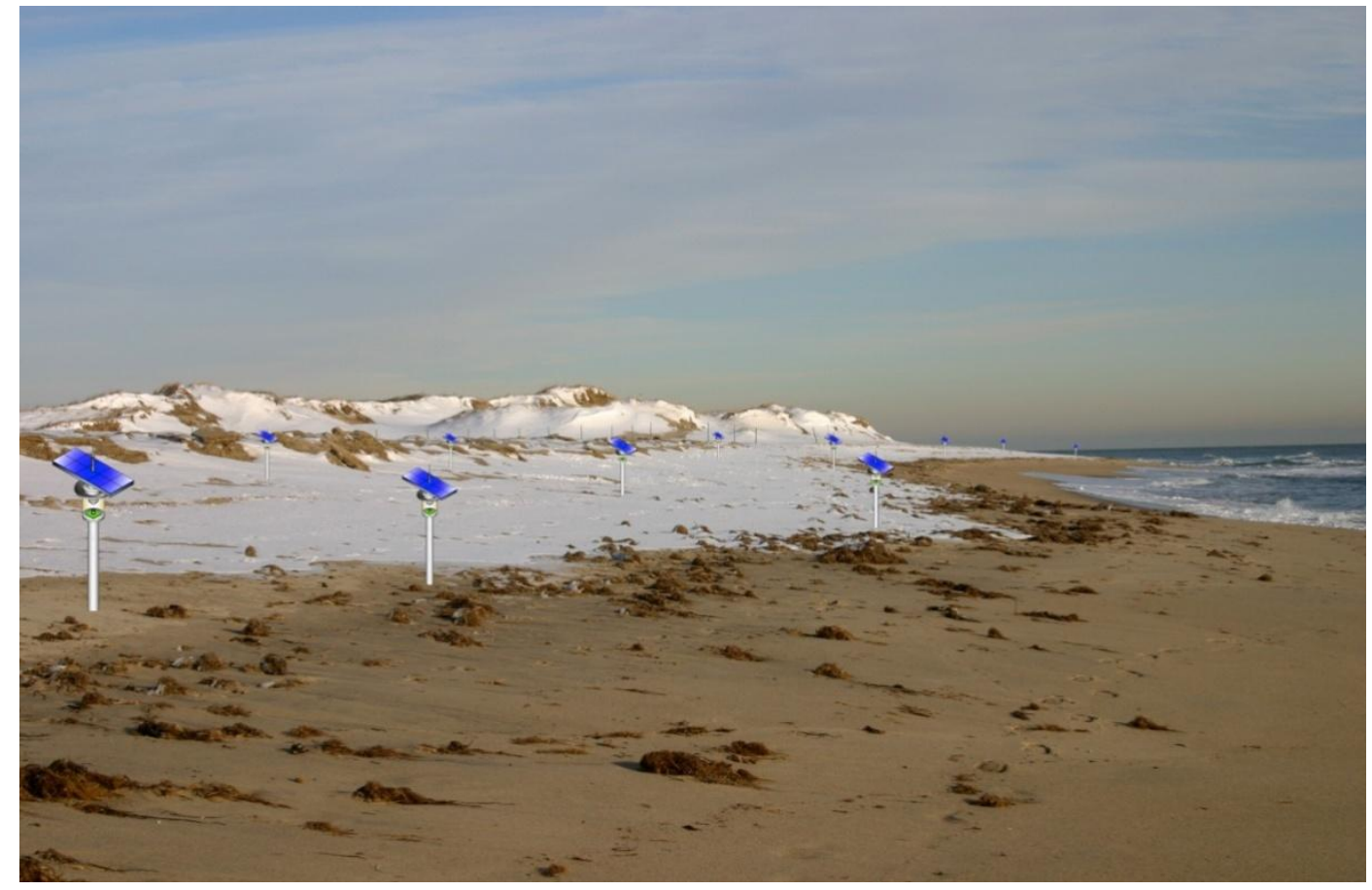

Figure 1. WVSN Application Deployment Scenario

The remainder of this chapter is as follows: Section 2 provides a background on video streaming over wireless sensor networks. Section 3 introduces the challenges and characteristics of routing algorithms for WVSN. Section 4 focuses on the survey of the state-of-the-art data routing techniques for video streaming application over WSNs. Section 5 explains performance evaluation criteria for different data routing techniques on WVSNs and proposes design metrics for their characterization. Section 6 presents a methodology to justify the performance of different video data routing techniques. Section 7 concludes the chapter.

\section{PRELIMINARIES FOR WVSN}

Video streaming in a WSN can be conceptualized as three cascaded components: video generation, video delivery, and video playback as shown in figure 2.

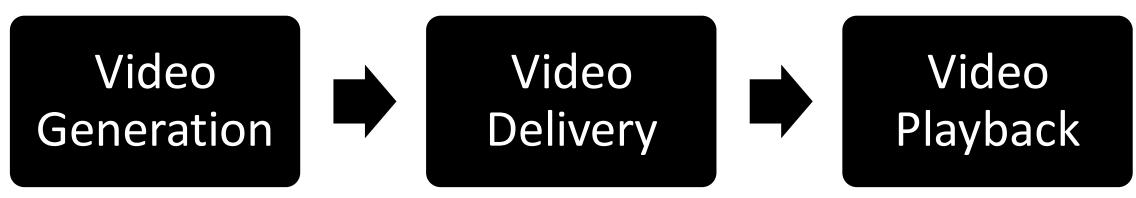

Figure 2. WVSN Decomposition 
In the following, we list critical design elements with their definitions and roles in each of the components.

\section{Video Generation}

○ In-Network Processing: Closely-positioned video cameras record highly correlated video content. In-network processing acts as a filter at the beginning of video recording to remove content redundancy. Localized information is shared by neighboring video nodes to coordinate video recording for a targeted area.

- Video Processing/Data Compression: To save energy for data transmission, video processing is used to extract relevant features of interest prior to transmission to minimize the total data transmission energy cost.

o Video Coding: Video coding is critical for success of video streaming. Many coding techniques have been developed supporting a variety of goals. For example, network coding is introduced to achieve high data transmission efficiency, multiple description coding (MDC) is used to achieve reliable data transmission and provide QoS guarantees.

\section{Video Delivery}

○ Bandwidth: Generally speaking, bandwidth is a resource that a link obtains to deliver the data over a communication link measured in bits per second. Bandwidth is also used as an indicator of link capacity. Congestion occurs if the potential load is larger than the link bandwidth. However, in wireless video networks, link capacity can only be viewed as a relaxed link load upper bound due to radio collisions; real link throughput is far less than the available capacity.

- Multihop Routing: Multihop routing refers to the use of intermediate nodes to propagate data from source to destination. Multihop routing reduces the cost deployment by eliminating wired infrastructure, but is practically limited by progressive degradation as hops increase.

- Multipath Routing: A path is a series of cascaded links between a source and a destination. Multipath routing defines the use of multiple paths between the same source and destination pair to deliver data. Multiple paths provide an option for increasing the capacity to deliver data to a destination if congestion at the source and destination can be managed and the interference among different paths can be controlled.

- Energy Efficient Routing: WSNs assume battery-powered operation perhaps with energy replenishment via harvesting technology. The depletion of battery power at individual node can result in broken links in the established network topology and influence the continuity of video data transmission. Energy-aware routing is necessary to achieve policies for managing energy depletion that can disrupt network connectivity and the video dissemination mission.

- Latency: Latency describes the delay of a data packet from source to destination. Streaming data is affected by both delay and delay jitter. The packets consisting of a video frame must all be correctly received at the destination before the playback of the frame. If the packet cannot be delivered within the deadline, this packet would be obsolete and useless. The data routing component has a goal of delivering packets on time while real time streaming is a requirement.

- Data Dissemination Pattern: Many practical sensor networks funnel data from leaf nodes in a tree structure to a single data sink. This model creates congestion and energy depletion in the region of the sink, especially for wireless nodes. Video sensing exacerbates this problem due to its data density. For more general-purpose design of video data routing, the data dissemination model is on demand to support multiple sources and multiple sinks. 


\section{Video Playback}

○ Playback Gap: This gap is defined as the period between initiating video download and the start of playback. Video streaming technology enables users begin playback prior to completing the full download. A local buffer is used to cache downloaded video, and successive frames are fetched from the buffer for playback. The larger the buffer, the more able the playback can accommodate delay variations (jitter) in the video transmission.

- Video Distortion: Video distortion defines the visual quality degradation due to the lost data.

Video delivery is a critical component for achieving video streaming over WSNs. The performance as quantified as quality of service (QoS) for video playback is highly depended on the video delivery techniques. Although video generation and playback are relevant topics, they are not the focus of this chapter. In the following section we introduce the characteristics and challenges for achieving routing in a WVSN.

\section{CHARACTERISTICS AND CHALLENGES FOR ROUTING IN A WVSN}

Routing is an essential component for video streaming in WVSNs. Routing deals with three basic issues: where to route the video data, how to satisfy the end-to-end delay requirement of the video streaming application and how to keep the network functional as long as possible under energy depletion. We label these three challenges as: Addressing, Resources, and QoS as described next.

\section{Addressing Challenge:}

Unlike IP (Internet Protocol) addressing which is hierarchical, sensor networks are typically configured with flat addressing that leverages locality. Assigning global IDs for each sensor node is not always a requirement. Moreover, a hierarchical model can introduce excessive overhead when most communications are neighbor-based. And in some cases, based on the characteristic and function of wireless video sensor network, there can be no need to distinguish one node from another by ID (e.g., Intanagonwiwat et al., 2000). Thus, the development of routing protocols based on IP address is not a requirement for a WVSN.

\section{Resource Constraint Challenge:}

A typical wireless sensor network consists of a large number of autonomous, inexpensive and simple sensor nodes that are powered by batteries. Although advances of circuit design have made substantial improvements for battery life in recent years, compared to desktops in wired networks, sensor nodes are still strictly constrained by energy. Such limitations have a great impact on communication ability and routing protocol design.

\section{QoS Requirement Challenge:}

Designing a routing protocol to ensure the sensed video data to be delivered reliably and timely while traversing a series of unpredictable wireless links is an ongoing research challenge.

Understanding the above challenges leads us to the creation of quantitative metrics for evaluating the success of existing approaches, which we describe next.

\section{STATE OF THE ART VIDEO ROUTING TECHINIQUES}

In this section we review the recent works focused on routing algorithm design for supporting video transmission in WVSN applications.

\subsection{Addressing Techniques}

\subsubsection{Content-Based Addressing}


As previously indicated, for many wireless sensor network applications, it is not practical nor a requirement to assign global IDs such as an IP address to each sensor node. Many investigators have recognized that the data flow within a sensor network can be characterized by the content of the data itself. A mapping between the sensor node's functionality and data content can be established during data transmission. By summarizing the data interests of participating sensor nodes, content-based routing is achieved (Intanagonwiwat et al., 2000; Guo et al., 2008; Carzaniga et al., 2004). In such a routing scheme, data-receiving nodes propagate data interests across the network in a publish-subscribe or push-pull model. Guo et al. (2008) provide an example of the 'push' method with the use of an application-specific property to distinguish different nodes. A node address is represented by a set node attributes defining its function in the application (e.g., 'with a light sensor,' 'measure rate,' etc.). Each data stream is prefixed by a structured description using typed language which defines a set of criteria for the destination node of the stream called predicates, such as 'nodes inside certain area,' 'node with certain brand.' The protocol establishes a hierarchical property-interest tree rooted at source node. The source node pushes the data stream onto the tree and thus the stream will flow to the node with the corresponding interest. Directed diffusion (Intanagonwiwat et al., 2000) is an example of content-based routing using the 'pull' method. Directed diffusion aims at diffusing data through sensor nodes by using a naming scheme for the data. A significant advantage of directed diffusion is the simplification of the programming abstraction and routing mechanism for propagating data to a data sink. Direct Diffusion suggests the use of attribute-value pairs for the data and queries the sensors in an on demand basis by using those pairs. In order to create a query, an interest is defined using a list of attribute-value pairs such as name, interval, duration, geographical area, etc. A sink broadcasts an interest through its neighbors. Each node receiving the interest broadcast caches the interest for later use. The nodes also have the ability to perform in-network data aggregation to merge similar interests. The cached interests are then used to compare the received data with the values in the interests. The interest entry also contains several gradient fields. A gradient is a reply link to a neighbor from which the interest was received and is characterized by a data rate, duration and expiration time derived from the received interest fields. Hence, by utilizing interest and gradients, paths are established between sink and sources. Several paths can be established so that one of them is selected by reinforcement. The sink resends the original interest message through the selected path with a smaller interval hence reinforces the source node on that path to send data more frequently. There are many variations proposed for efficient wireless sensor network data routing based on Directed Diffusion.

GEBR (Li et al., 2007) expand the concept of Directed Diffusion to provide global energy balancing and real-time routing for video data transmission. Their path formation process is nearly identical to that of directed diffusion; however, they introduce node energy as a criterion for data transmission and path reinforcement.

Wang et al. (2007) propose synchronized pipelined transmission for video data streaming. Unlike Directed Diffusion which floods interest message to explore the optimal path, the route discovery process in uses a probabilistic method. The source node periodically sends out route probing packets. 
The probing packets are randomly relayed to a neighbor of the current hop until they reach the subscriber node. When the predefined route-probing timer expires, the subscriber node calculates the optimal path based on all received probing packets. Although this process can largely eliminate the data load created by the path exploration stage, it performs poorly when the network is relatively large and the source and destination nodes are scarce and far from each other.

Li et al. (2008) provide a multipath data delivery solution to deal with the challenge of delay control in video transmission applications with another expansion of directed diffusion. Instead of using the metric of transmission time, the scheme uses a weighted metric that captures delay, interference and throughput. A timestamp is given to both interest message and exploration data. The sink station chooses exploration data whose timestamp is within a predefined threshold to satisfy the delay constraint as compared to the interest message's timestamp. The sink tags the senders of these qualified exploration data messages into the reinforcement path candidate pool. The reinforcement scheme in this solution supports the set-up of multiple disjoint paths. However, the length of the path set-up period is largely dependent on the compound metric it adopts and subject to variable delay. Furthermore, the data supporting the adopted metric is difficult to obtain. For example, the measurement of SNR needs the power level of three terms: noise, interference, and signal strength. The author does not provide the method to measure such terms. A simpler metric that can be directly obtained or estimated by the network layer is more preferable and flexible.

From the above exploration, we find that there are three major problems existing in the current content-based video routing technique for wireless sensor networks. First, all of the current techniques are application-dependant addressing schemes. It is difficult to port data interest from one application to another unless the two applications are similar. Second, the content-based routing schemes utilize a route exploration stage using route exploration data. The motivation for this approach is to simulate data transmission and thus select what might be an optimal route. However, in video streaming applications, the prevailing conditions during route exploration can be substantially different from when video data are in transmission. In other words, a good route obtained during a data exploration stage will not necessarily be the good route during a data transmission stage. Thus, a specially designed exploration stage is required to find a good video streaming route for WVSNs. Third, we lack the control of path selection involving overused nodes. It is very likely that multiple data paths will share common nodes since these paths become reinforced. These nodes soon become overloaded and compromise the video data transmission.

\subsubsection{Location-Based Addressing Techniques}

The second group of addressing schemes is location-based. A number of proposed routing schemes fall into this category. Since most WSNs are comprised of nodes deployed in a known area, they have proper coordinates established that can be used to assist routing. The distance between the source node and sink node in real world can be used as a proxy for the energy cost for data delivery. There are two kinds of location-based routing strategies. The first one we call real coordinate routing. GPSR (Karp \& Kung, 2000) is one such example. In real coordinate routing, each node uses geometric distance as the routing metric. This strategy establishes coordinates for each node based on its absolute (Cartesian) location. By obtaining the location information of a destination node and its neighbor nodes, senders always forward a packet to a neighbor with a shorter distance to the destination (a greedy technique). 
Real coordinate routing suffers from well-known dead-end problem especially in a sparse network or one with physical obstructions. The second strategy is called virtual coordinate routing (Rao et al., 2003; Newsome \& Song, 2003; Zhao et al., 2007). This routing strategy applies routing metrics to reflect the relative location of the sensor nodes within the network instead of using absolute coordinates. Zhao et al. (2007) propose a method in which individual node constructs a vector with elements corresponding to the hop distance to the set of pre-established landmarks. This vector is exactly the virtual coordinate of the node. The routing process is identical to GPSR (a greedy formula) except that it uses a more elaborate distance function instead of the geographic distance. Virtual coordinate routing performs well for the dead-end problem in sparse scenarios and it reduces the hardware requirements of sensor nodes. However, the virtual coordinate setup process is not easy and is energy consuming. Thus most recent works in location-based routing continue to use real coordinates.

Cosma et al. (2006) have an interesting application of location-based routing for video streaming. This paper is not a complete solution for video transmission over wireless sensor networks but introduces a topology extraction protocol using video cameras equipped on each sensor node. There are two steps to achieve the topology extraction. First, a central node/server or gateway floods routing messages over the network and every node records routing information. After a path set-up phase, every node in the network captures an image using its video camera, and passes the image through to the central node/server. This node then performs image registration to extract the topology and location of each sensor node. The result is analogous to a bird's-eye view of the global topology of the system. The authors further suggest that the global topology can be optimized for path routing and energy conservation. This scheme is creative but impractical at present. Image registration of a large number of disparate images is complex, time consuming, and potentially performed with sparseness of view. It is also likely that the extracted topology has significant error due to the limited camera resolutions, focal lengths, and fields of view.

DGR (Chen et al., 2007) is a mechanism proposed to transmit real-time video. The idea of DGR is to construct an application-specific number of multiple disjointed paths for a video node to transmit parallel FEC-protected H.26L real-time video streams over a bandwidth-limited, unreliable networking environment. Unlike traditional location-based routing algorithms using greedy routing schemes (Karp \& Kung, 2000; Yu et al., 2001) the author introduces a concept of "deviation angle" to spread the paths in all directions by the side of the line-proximity of the source and sink nodes. It implies that packets along some paths are likely to be forwarded to a neighbor that is a greater distance from the sink. To deal with problems with route coupling (Pearlman et al., 2000) caused by interference between packets transmitted over different paths, the authors separate physical paths as far as possible. When a node receives a path set-up packet, it calculates its virtual coordinate based on the location of the upstream message sender, destination, and itself. The origin of the virtual coordinate is the upstream node's location and a reference line ( $\mathrm{x}$-axis) is between upstream node and sink node. The angle between the $\mathrm{x}$ axis and the line segment of the receiving node and the upstream node is then obtained. The upstream node then chooses the node whose angle is least different from the "deviation angle" as the next hop. Deviation angle is controlled by a function with respect to hop count to ensure the path will definitely go back to the sink. DGR uses node location to identify different sensor nodes. Instead of using pure location for routing decision, DGR introduces deviation angle-controlled routing to find detours. This idea is efficient for establishing multiply separated paths from source to sink. The video data can then be subdivided into multiple streams and transmitted through multiple disjoint paths to the sink.

The TPGF routing protocol (Shu et al. 2008) is another example of a greedy location-based scheme. In order to solve the hole-bypassing problem (Fang et al, 2004; Yu et al., 2007; Jia et al., 2007), TPGR 
proposes "step back and mark" process to explore possible paths to the base station and guarantees to find a route to the destination as if one exists. This protocol is designed to execute multiple times to find multiple disjoint paths from source node to sink node. However, unlike the scheme adopted by Chen et al. (2007) which introduces a way to separate paths as far as possible, on the contrary, this scheme put these paths as close as possible to the centerline which can cause very severe path coupling problems.

According to the above analysis, location-based routing schemes are application independent. Moreover, the information required for data routing is simple and localized without necessary knowledge of the global network topology. But some variants are not entirely practical. For example, most location-based routing exploits GPS data that is often unavailable due to cost or indoor locations. Another problem for location-based routing is to deal with network holes. An efficient hole-bypassing algorithm is very important for location-based data routing over WVSNs.

\subsubsection{Hierarchical Addressing Techniques}

Yet another routing scheme is based on hierarchical addressing. The basic idea of hierarchical addressing and routing is to group sensor nodes into multiple clusters based on some assignment criteria. A cluster "head" is selected to coordinate communications within the cluster and to any nodes corresponding to other clusters. LEACH (Heinzelman et al., 2000) is a milestone protocol in this area and it inspires a large number of hierarchical routing protocols for wireless sensor network. The idea of LEACH is to form clusters based on radio signal strength and to use local cluster heads as routers to the sink. This scheme saves energy by simplifying routing in a locality and managing the propagation of data that must traverse multiple clusters. The optimal number of clusterheads is estimated to be $5 \%$ of the total number of nodes. All the data processing such as data fusion and aggregation is local to the cluster. The assignment of clusterhead is rotated in order to share the energy burden of this function.

Akkaya and Younis (2003) provide a three tier network architecture to route data as illustrated in Figure 3. Before network operation is established, sensor nodes are grouped into clusters. Each cluster has a gateway node. Sensor nodes only route data to the gateway nodes, and gateway nodes are responsible for routing data to the central command node. The sensor nodes do not require globally unique IDs. The path setup process is a centralized scheme. The cluster's gateway node is assumed to know the cluster's topology and link state between any two nodes inside cluster. The idea proposed is to find a detour path to the gateway instead of transmitting data directly. However, the authors neglect to consider that all the sensor nodes of a cluster are within the radio range of the cluster head and are thus potential interferers. Changing the route without changing the radio range can cause severe interference.

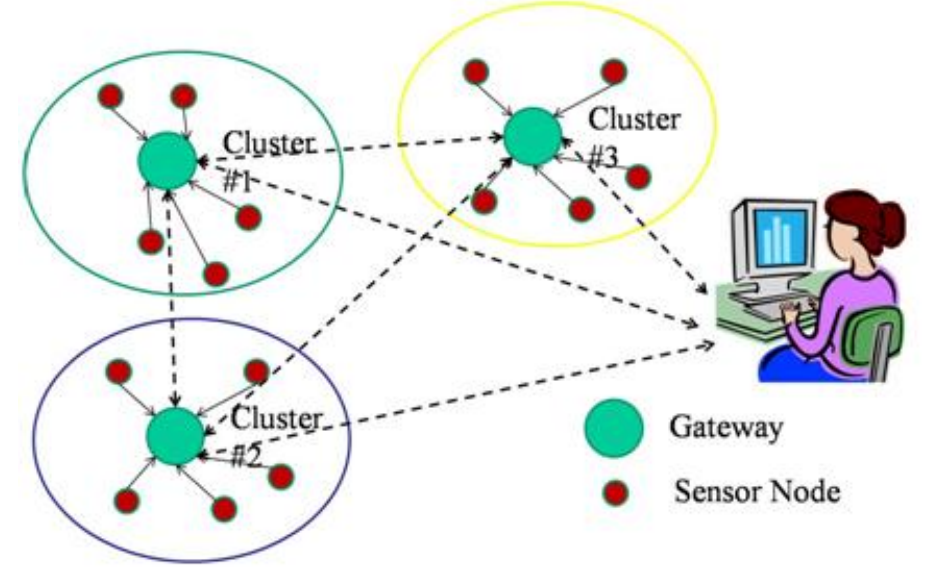

Figure 3. Three Tier Network (Adapted from (Akkaya \& Younis, 2003)) 
Politis et al. (2008) describe another hierarchical video data routing scheme. The network architecture setup is a slight modification of architecture of LEACH. Instead of using a direct link between a cluster head and base station for data collection, cluster heads are allowed to establish links to each other. Hence, a video sensor node can select a number of available paths through other cluster heads in order to transmit its data to the base station. This modification decreases the transmission power of a clusterhead for shorter-range communication and saves energy. This paper can be viewed as a complementary work of Akkaya and Younis (2003). Instead of addressing routing inside a cluster, Politis et al. propose a scheme to address routing from clusterhead to a base station. The algorithm is adapted from the work of Chen et al. (2004) and it uses a centralized algorithm based on the knowledge of the network topology, link capacity and link delay. Unfortunately, the scalability of such routing algorithm is not sufficiently addressed.

Besides the communication management benefit, another inspiration to use hierarchical network architecture for video data transmission is that such architecture is efficient for redundant data removal. High data rates of a video stream inevitably will cause rapid energy consumption by sensor nodes. To avoid node failure and network disruption due to battery depletion, an aggregation-driven routing scheme is proposed. In a hierarchical network, nodes from lower level send their data to higher-level nodes for data aggregation. Nodes at a higher level are then responsible for comparing and removing redundant data from each stream and compressing the data before sending them to their upper-level controllers. Navda et al. (2006) propose one such routing scheme. The basic idea is to merge multiple flows at early stage of data transmission and form spatially separated paths to minimize inter-path radio interference. The root node first floods a message to setup a spanning tree. Source nodes attempt to send data to the neighbors of the root through nodes which are not carrying any flows and do not have contending transmitters in their radio range. If the node cannot find such path, it will route to the nearest node that carries the fewest flows. Unlike traditional schemes which route data through disjoint paths, this scheme tries to merge data at an early stage of the data transmission and form spatially independent routing paths.

From the above analysis, the main advantage for building a hierarchical network topology is for data fusion and data transmission management. However, the data fusion costs for video are not very carefully studied in this prior work. According to Liu \& Das (2006), the fusion cost of video can be equivalent to that of transmission. The shortcoming of hierarchical network organization is the imbalanced network load distribution across participating nodes. Such a network would result in unfair resource consumption for different nodes in different layers.

\subsubsection{Global ID Routing Attempts}

The absence of globally unique IDs hinders the integration of WSNs and IP-based networks. In order to solve this problem, schemes have been investigated to assign unique network-wide IDs (OuldAhmed-Vall et al. 2005). However, these ideas face the risk of incompatibility with the established standards of the Internet. Another approach is via IPv6; a sensor can concatenate its cluster ID with its own MAC address to create a full IPv6 address. However the 16 byte address field of IPv6 potentially introduces excessive overhead in each sensor data packet, but in this way, the existing Internet solution for video transmission can be adapted to achieve video transmission over a wireless sensor network.

\subsection{Energy-Efficient Routing Techniques}


As stated at the beginning of this chapter, energy consumption is always the primary concern of wireless sensor network application design. This section considers recent efforts in energy-efficient routing.

The energy saving idea from GEBR (Li et al., 2007) is to send data through the path of fewest hops and most longevity. The longevity of a path is measured with something called Minimum-Path-Energy (MPE), which is the minimum energy of all the nodes along a path. The interest message generated by a sink destination contains hop count requirement, MPE value, and path length. When a source node receives a set of interest messages, it calculates the maximum value of the MPE from different paths whose path length is smaller than the hop count requirement. The hop count requirement is a proxy for a real-time streaming requirement. The authors assume that a path with fewer hops will yield the lowest data transmission delay. Afterwards, the source station sends exploration data that contains the maximum MPE value (BMPE value). The node only forwards the exploration data to its upper stream node if the neighbor's energy is larger than the BMPE value. This process ensures that exploration data only goes through the most survivable path and balances the traffic load. However, the pure BMPEbased routing does not consider problems with packet collision. If multiple source and sink nodes exist, it is very likely that the BMPE path will be heavily loaded or even be badly interfered with by other BMPE paths resulting in significant packet losses, delay, and energy waste. Figures 4 and 5 illustrate examples of BMPE generation and optimal path selection:

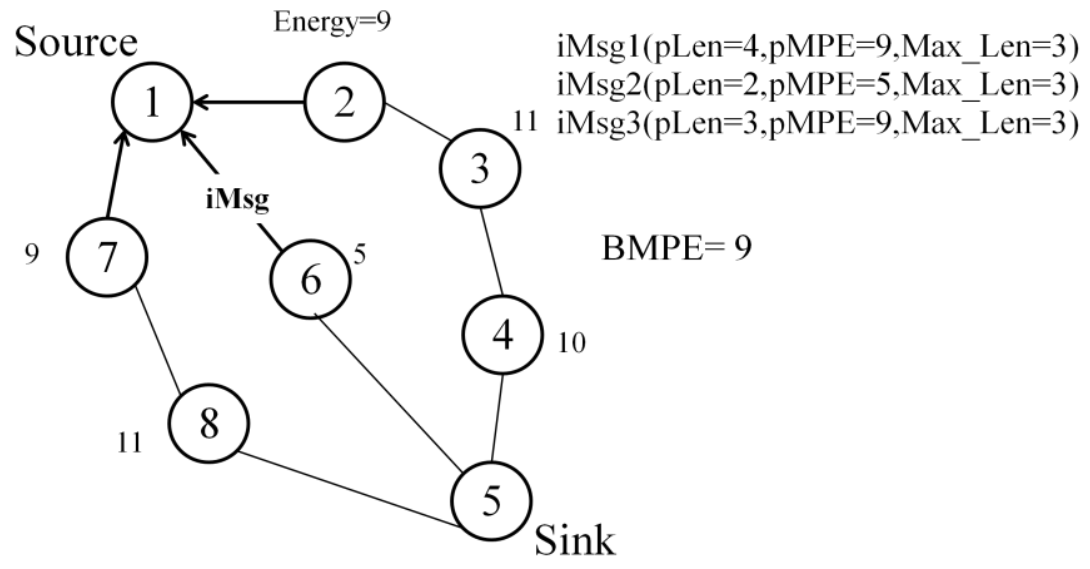

Figure 4. BMPE Generation (Adapted from (Li et al., 2007))

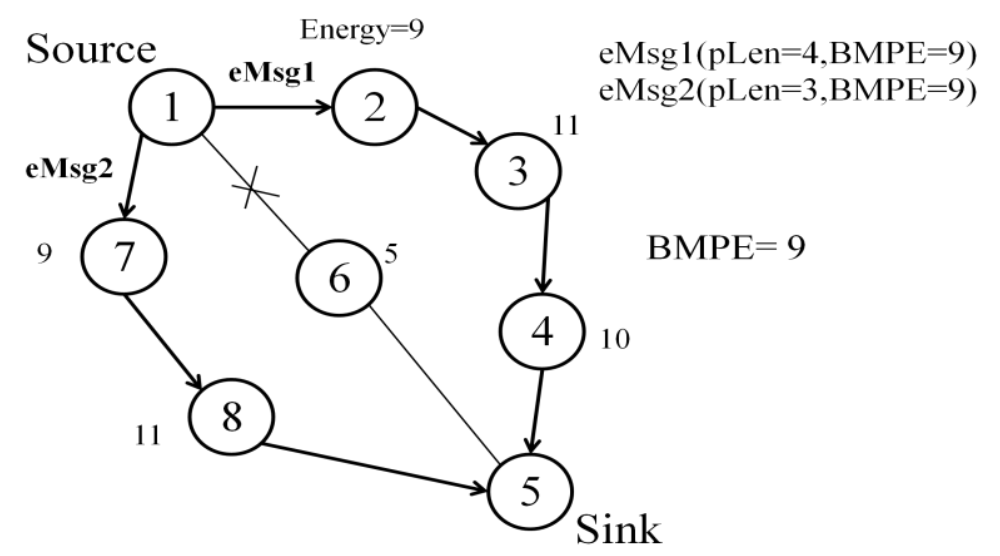

Figure 5. Optimal Path Selection (Adapted from (Li et al., 2007)) 
Wang et al. (2007) consider energy conservation through the reduction of packet retransmissions in the presence of node failures. A synchronized and pipelined transmission scheme is proposed with flow control. They use a secondary buffer to ensure the maximum retransmission distance is not more than the equivalent of twice the size of the failed node's buffer. However, it is not clear how well this scheme scales under their synchronization requirement. The flooding of synchronization messages will not be efficient for large networks. Instead, synchronizing node for a particular route seems more feasible and efficient. Also, buffering packets at the neighboring nodes is a good idea; however, to the means to update these secondary buffer data according to the update of the primary buffer data is not described.

Cosma et al. (2006) propose that every node maintains a record of their neighbors' energy level and hop count to the server. Any node with a relatively high energy $(>20 \%)$ will be in the candidate set for next hop during routing. The candidate with smallest hop count will be chosen as the next hop. This solution, although more conformal to energy fairness on a per hop basis, does not prevent instantaneous overuse of a path. Moreover, for long-lived video streams, there can be significant change in energy levels for nodes participating in multihop routing. How to adapt to short-term energy change and contention for shared paths is not considered.

Based on the first-order radio model (Heinzelman et al., 2000; Shin et al., 2006; Fang et al., 2004), Shu et al. (2008) derive the node energy consumption rate as a function of data rate and radio transmission range. The goal here is to manipulate the radio transmission range to satisfy the energy consumption constraint to achieve a target network lifetime. Results indicate that a greater transmission range leads to lower latency. A node will prefer to use the maximum radio range if the corresponding node energy consumption rate is lower than the expected rate. The radio range and energy consumption rate are computed based on two critical terms: the energy cost to power the transmitter circuitry to send one bit, and the energy cost for transmitter amplifier to send one bit. These two terms are difficult to measure; inaccuracies will interfere with the success of the routing algorithm.

Politis et al. (2008) introduce the clusterhead energy consumption model. The authors propose a packet-scheduling algorithm that allows a source node to drop packets queued for transmission in order to avoid downstream congestion. This is achieved considering the residual energy of the clusterheads on the path to the destination.

Akkaya \& Younis (2003) address energy efficient routing in the context of a single cluster. They assume that all sensor nodes in a cluster are within the radio range of its associated gateway. A link cost function is defined based on the consideration of delay, residual energy, distance, and other factors. The gateway node is assumed to know all link states; Dijkstra's algorithm is applied to find the least cost path between sensor nodes and gateways. The gateway continuously monitors the available energy level of every sensor node active in data processing, sensing, or relaying. Rerouting is triggered by the depletion of energy of an active node. But the details of node residue energy monitoring and energy metrics are not disclosed in this paper.

In summary, we find some practical limitations of many of the exiting energy-constrained routing techniques. Either there is no clearly established energy metrics or the existing metrics are difficult to obtain robust measurements. Designing a practical energy consumption model and residual energy monitoring protocol would be very helpful for energy-efficient video data routing.

\subsection{QoS Techniques}


Video and audio data transmission requires certain quality of service (QoS) achievement in a WVSN, especially when streamed continuously. For example, a streamed video must deliver each frame to the user on-time to achieve continuous playback. Unfortunately, there are many uncertainties in WVSNs that can cause significant delay that diminish video playback quality. In the following we discuss techniques that have been adopted to deal with such challenges.

Li et al. (2008) propose to deliver MDC-c--coded data through multiple selected paths to overcome packet loss due to network congestion and transmission delay. Cross-layer design and disjoint routing path selection are considered to provide better QoS. During the path exploration period, the algorithm tags identified paths into a pool and sorts them in an ascending order according to the path length. The first $\mathrm{N}$ shortest paths that satisfy the data delivery cost constraint are chosen to be candidate routing path. In order to find multiple reliable paths from the source to sink, the sink chooses the first $\mathrm{N}$ shortest paths based on cost. $\mathrm{N}$ is chosen to be a slight larger than the required number of paths for the transmission, since some candidate paths may not be reinforced if disjoint nodes cannot be found or the delay exceeds the playback deadline. During the path reinforcement stage, if two nodes happen to reinforce a same node, the second reinforcement will be invalid to guarantee disjoint path selection and avoiding loop generation. To deal with bottleneck problem shown in Figure 6, Li et al. (2008) eliminate the existence of bottleneck links through a deployment density control.

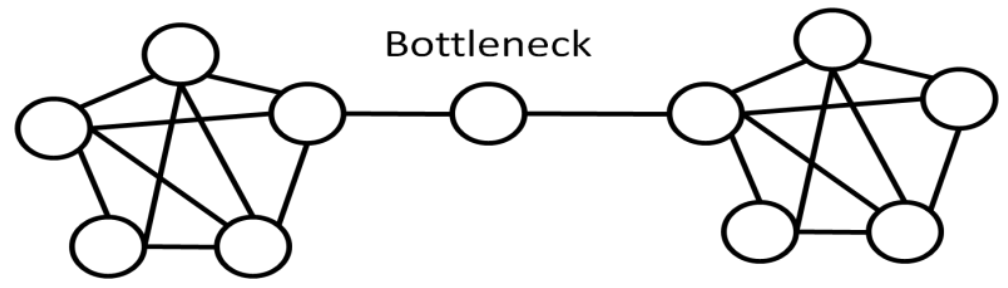

Figure 6. Node A is the Bottleneck (Adapted from (Li et al., 2008))

DGR (Chen et al., 2007) uses multipath transmission to achieve low latency. A FEC coding scheme is used to recover data due to packet loss or data corruption. Although multiple path transmission can expand available bandwidth, it is not clear how data path merging is achieved which can result in severe data contention at the destination.

Base on TPGR (Shu et al., 2008), L. Zhang et al. (2008) uses a multi-priority multipath selection scheme for video streaming for WSNs. In this technique, a priority index is assigned to different paths based on end-to-end delay. Streams are identified and prioritized based on their ability to monitor an event. Streams are also split into audio and video components. A mapping scheme is implemented to assign a higher priority path to a higher priority stream. A shortcoming of this scheme is that it relies on direct paths from source to sink and does not fully address the path interference problem.

Akkaya and Younis (2003) propose a link cost function that considers link delay. The idea is to adjust the bandwidth split ratio $r$ to let the average end-to-end delay satisfy the delay requirement. The average delay estimation is based on an implied assumption that each packet could be delivered to the destination through multiple hops without any corruption or loss. If we consider packet collision or loss, we expect that the average delay of each packet will increase beyond their model's prediction and the bandwidth split ratio value $r$.

Politis et al. (2008) propose to transmit the most important video packets through multiple paths to the destination to achieve a QoS target. A video distortion model presented by Politis et al. (2007) is 
applied to estimate the importance of different encoded video packets of the H.264/AVC stream. The algorithm developed by Fang et al. (2004) is used to find multiple paths from source to destination that can satisfy the video bandwidth requirement. A baseline packet-scheduling algorithm is introduced to manage the transmission by dropping excessive video traffic based on the packet importance. For further improvement, the authors developed a packet-scheduling scheme that factors the residual energy of cluster heads in the algorithm. This approach can reduce the distortion of the decoded video sequence by deciding which and how many packets will be dropped according to transmission rate limitations and power failure of the nodes prior to transmission. Politis et al. (2008) borrow the multipath formation scheme developed by Chen et al. (2004). The path formation algorithm considers two factors: end-toend delay and aggregate bandwidth. This scheme guarantees to find multiple paths from source to destination that satisfy the bandwidth and delay requirements. However, due to uncertainty of wireless channels, the actual throughput of the multipath is usually much less than the aggregate value. A limitation of this idea is that it is only applicable to single source-destination pairs. An additional limitation is the reliance of the distortion-reduction algorithm on a specific video encoding scheme.

A rate-based flow control algorithm called doubling and reducing-by-half is proposed by Navada et al. (2006). By monitoring the throughput of per flow at the root, the source is allowed to double the load of the flow until the throughput of other flows is influenced and dropped under a threshold. Thereafter, the source node reduces load in the next step to half of the previous incremental load until throughputs per flow match again. Packet scheduling based on packet delay requirements and flow priority is proposed to promote performance. Also, early dropping of packets is used to deal with anticipated congestion. Unfortunately, these schemes are not fully analyzed to demonstrate their practical applicability.

Chai and Ye (2007) adapt Internet streaming models the wireless mesh context. Each node is assumed to have a unique IP address. Data consumers contact a central server for authorization and channel setup. The central server coordinates different video servers to stream video data to the corresponding users. A media transfer server working at background is responsible for converting the various multimedia file formats to the internal streaming file format suitable for reading and indexing by the delivery server and uploading data to the distributed file systems or data storage center. The authors adapt RTSP/RTP protocols used in Internet video streaming to stream the video data over wireless mesh network. The only difference is that the underlying transmission mode of RTP packet is replaced by TCP connection to overcome the high error rate and bandwidth fluctuation of the wireless channel. Authorization process and packet scheduling algorithm for multi-users is introduced to solve the challenge brought by RTP-on-TCP transmission such as robust and security connection problems, delay jitter problems and system blocking problems. Non-important packets can be dropped before transmission to avoid potential network congestion and improve network performance.

In reviewing recent QoS management techniques for video delivery in WSNs, three main techniques emerge as best candidates for adoption:

1. Multipath Transmission: This technique is used to expand the available network capacity through multiple links. Existing proposals can benefit by additional models for path interference and wireless data transmission.

2. Controlled Packet Scheduling: This technique is used to predict and reduce data congestion within a network. Priority-based packet scheduling is favored to achieve required QoS.

3. Coding: Video coding is used to overcome the corruption of data during transmission and to mitigate the frequency of retransmissions, thus reducing data volume and associated energy consumption due to transmission. 


\section{METRICS AND PERFORMANCE EVALUATION CRITERIA}

We seek a good design for video data routing. A design must consider WSN video application requirements and yield a reasonable tradeoff of performance based on current technologies limitations (e.g., battery capacity, video encoding, and radios). In this section we propose a canonical set of metrics on which we can base design decisions and performance evaluation for future designs.

\section{Resource Conservation Performance:}

As we know, WVSNs are resource-constrained. Applications built on WVSNs are limited by bandwidth, battery energy, available channels, etc. Performance evaluation in this dimension will indicate that how good the routing technique will perform to save limited network resources. The following metrics define resource conservation performance and are adopted from a survey of prior work:

- Number of Channels: Number of channels utilized for wireless communication.

- Residual Energy: Instantaneous battery energy at a sensor node (Joules)

- Circuit Power: Instantaneous energy consumption at a sensor node.

\section{Path Formation Performance:}

There are two metrics related to routing: path setup delay and path setup flexibility. The former indicates how quickly an algorithm will respond to a request to send data and the latter indicate the reusability of a path setup by the algorithm. The two metrics are defined as follows:

- Path Setup Delay: The time period for a data path to be found and ready for data transmission.

- Path Setup Flexibility: Assessment of the adaptability of the path instance especially for related data transmission requirements.

\section{Data Delivery Performance:}

Data delivery performance is one of the most important characterizations of WVSN routing designs. This performance indicates how well the data will be transmitted along the data path. Two critical terms are evaluated:

End-to-End Delay: The time to send one packet from a source to a destination

- Metrics:

○ Hop Count: Total number of hops from source to destination, generally speaking, a larger hop count corresponds to a larger delay.

- Propagation Time: Time for a packet transmission from source to destination.

- ETX (Expected data transmission time): The expected number of transmissions for a successful packet transit. Generally speaking, the larger the value the higher the delay.

- Data Path Capacity: The maximum data rate can be achieved in the absence of any cross traffic. This capacity is equivalent to the capacity of bottleneck link along the path

- Packet Queuing Delay: The average queuing delay of a packet on a path.

○ Throughput: Average number of packets traversing a path per unit time. 
Transmission Interference: Interference caused by data transmission on adjacent links.

- Metrics:

○ Link Failure Probability: the likelihood of a link to fail.

O SNR: signal to noise ratio

○ BER: bit error rate

\section{Network Performance:}

Network performance defines aggregate performance of all data paths in a network. In much of the related work we find that performance evaluation focuses on single path performance rather than aggregate performance. Network performance evaluation considers interactions among different data paths and indicates the global performance of the routing protocol. We propose the following metrics for network performance measurement:

- Number of active paths: How many video streams can be active simultaneously in the network. This metric reflects the capability of the routing algorithm. A good routing algorithm will support as many video streams as possible with relatively low overhead and energy cost.

- Average throughput per path: This defines the expected number of packets that are delivered per unit time for a path. This metric is a key benchmark of performance for a routing algorithm. If the average path throughput is higher than the threshold requirement of the WVSN application, then the algorithm is expected to perform well in practical application.

- Data Transmission Cost: The inverse of throughput. This metric can also involve energy costs.

- Data Collection Cost: Due to the data path throughput requirement, an upper bound for the path length will exist that makes the network base station unreachable with a certain probability. One possible solution is to deploy mobile gateways to gather data for the base station with certain deployment cost. Data collection cost is the total of the mobile gateway deployment costs.

- Data Transmission Cost: This term is defined as the total energy consumption for carrying a video stream on a certain path. It can be measured using the total active time of the sensor nodes along the path.

- Network Operation Cost: This is a measurement of total cost for delivering video data from sources to their destinations from the view of the network. This metric is usually a weighted combination of data transmission cost and data collection cost.

\section{RECENT RESEARCH RESULTS}

We have developed a performance evaluation framework for assessing the performance of video delivery in WVSN, which is described next.

We measure the performance of the video delivery based upon the network operation cost and the achieved QoS. Network operation cost is an abstract term that captures the cost of data transmission and data gathering. Designers using our following performance evaluation framework can adapt this metric to local conditions. We select throughput as our QoS metric. We select the throughput as our QoS metric due to that we believe many existing video delivery QoS requirements could be projected to this metric. Moreover, there are two basic QoS requirements for video delivery applications, timeliness and reliability. Timeliness requires that packets be delivered as promptly as possible. This requirement can be implemented by designing a routing algorithm with prioritized packet scheduling scheme to provide a lower bound of throughput satisfying the delay constraint of the application. The higher the video delivery throughput the more likely the application will deliver data on time. As a result, a timeliness requirement can be projected to our throughput requirement. A reliability algorithm leverages channel 
diversity to overcome packet loss while increasing the data redundancy by exploiting multi-path transmission. The data loss constraint in this kind of application can also be translated to throughput constraint. We can maximize the data redundancy to recover lost packets by designing a routing algorithm that achieves the maximum aggregate throughput at the destination. Such conversion enables us to measure the performance of these applications with our QoS metric.

Although achieving high QoS with less cost is an implied objective for many video delivery applications, most existing efforts focus on the optimization of either the cost or the QoS. A balanced consideration on both sides is not well presented. We find that the two design factors are indeed closely related to each other. Let us consider the network operation cost unit; it can be expressed as "cost/second". We can rewrite this unit as follows:

$$
\text { cost } / \text { second }=(\operatorname{cost} / \text { packet }) *(\text { packet/second })
$$

The first term on the right side is the average data delivery cost for each packet, the second term on the right side is the throughput of the whole network, which can be used as a QoS indicator. If we fix the network operation cost, we can plot the data delivery cost and network throughput as shown in Figure 7:

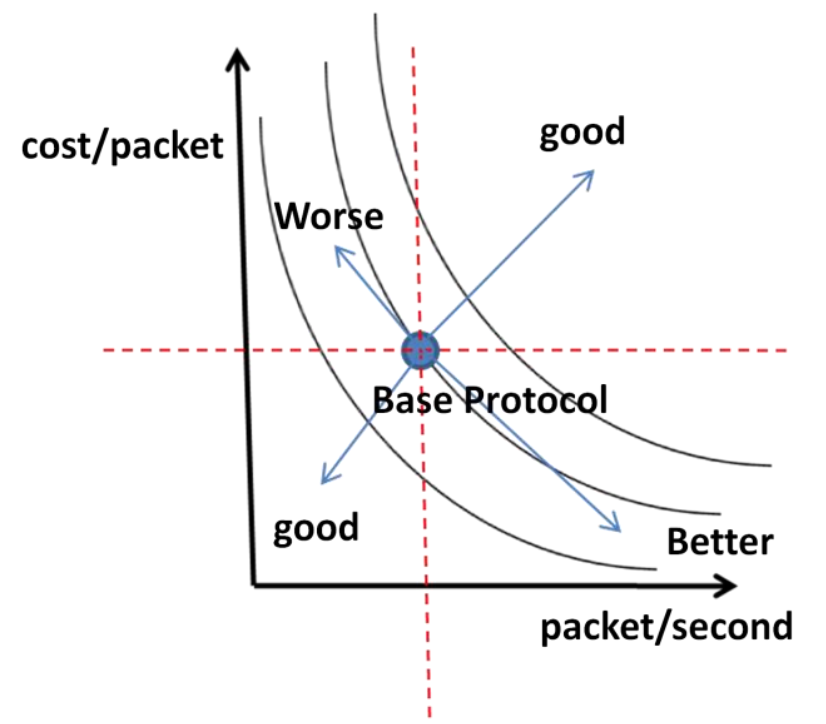

Figure 7. Video Data Routing Algorithm Performance Evaluation

The different protocols will achieve different performance nodes along the black curve. These curves correspond to different network operation costs. Given a location of the performance node for a base protocol as shown above, we can divide the graph into four quadrants. If a new protocol performance node lies in the upper left quadrant, we can immediately conclude that such protocol is even worse than the base protocol since the performance in this quadrant requires higher packet delivery cost but achieves lower network throughput. If the protocol performance node is in the upper right or lower left quadrant, we say that the new protocol is at least as good as the base protocol since these protocols achieve high throughput at high cost or low throughput at low cost. If the protocol performance node is in the lower right quadrant, we can tell that this protocol is better than the base protocol. The new protocol achieves higher throughput with lower packet delivery cost. Figure 7 is called constellation graph of video data routing protocol. No matter how many routing techniques you would like to compare for the application, as long as you have the values of the two metrics of each routing technique and plot its constellations, a simple glance of such graph will assess the performance of each technique. 


\section{CONCLUSION}

Based on the above analysis of the pros and cons of the state-of-the-art in video data routing in WVSN, we envision a series of design requirements for improved video routing protocol to deal with the proposed three basic challenges. These are:

\section{Addressing Challenge:}

The addressing scheme is better to be application independent. For example, content-based routing is a good idea to solve node identity problem but it is difficult to port data interest for one application to another unless the two applications are similar. Application-specific interest messages limited the commonality of these routing protocols. A better solution is to design an adaptive architecture for various applications. However, such interest generalization task requires extensive effort to explore the similarity among different potential applications.

\section{Energy Challenge:}

Energy conservation is a critical design challenge for video data routing. A path formation algorithm needs to consider energy use as a control parameter. Unfortunately support for accurate network-wise energy status measurement and consumption models are not well defined.

\section{QoS Challenge:}

QoS is an important design factor for video routing over wireless sensor networks. The design in this space focuses on transmitting data on-time using multipath and packet scheduling schemes to avoid network congestion and data loss. However, most of the previous work focuses on simple scenarios, a comprehensive study of multi-source multi-destination with unsynchronized data transmission must be conducted to understand the efficiency of any protocol. Furthermore, in wireless sensor networks, bandwidth and channel resources are limited. A simple addition of bandwidth of multiple paths is only the relaxed upper bound of the end-end throughput. Due to the severe inter-path and intra-path interference and network congestion, the actual delay and throughput can be quite different that one is found in an isolated path. Most previous works suggest the establishment of disjoint paths to ease the problem. The relationship of the packet collision probability, the path length, path number and path positions needs to be carefully studied to guide the path formation design. The use of path hop count as a reflection of potential packet delay is insufficient. A new path metric, which considers the path length, traffic load and queuing delay, would have strong potential for path formation.

Based on the suggestions above, we are exploring routing protocols to balance the outlined design requirements. We believe the new routing protocol must have a practical dynamic source-to-sink path formation scheme to isolate video streams, preventing intersection and collision, such a scheme could achieve full-capability live video streaming within a WSN while balancing the need for energy conservation via load balancing, in-network localized computation and path selection collaboration. New practical wireless data delivery models need to be introduced and with the help of such models, the protocol can derive the path formation elements, including number of stream per destination/video, network wide stream support capacity, and path length bound based on general requirement of video transmission QoS. These design elements/constraints could be computed at runtime during the video transmission in order to achieve a dynamic video data routing and scheduling.

So far, the routing protocol design we have discussed above is focused on traditional wireless sensor network where the wireless channel can only have limited bandwidth and can be easily interfered by other radio radiations. However, with the development of wireless technology we believe that this 
design bottleneck can be well addressed in the near future. Some researchers have attempted to incorporate CDMA/FDMA technology to address the interference problems. Some references in this area can be found in (Sohrabi et al. 2000; Caccamo et al. 2002; Liu et al. 2003).

\section{References}

[1] Akkaya, K., \& Younis, M. (2005). A Survey of Routing Protocols in Wireless Sensor Networks. Elsevier Ad Hoc Network Journal, 1(3), 325-349.

[2] Akkaya, K., \& Younis, M. (2003, May). An energy-aware QoS routing protocol for wireless sensor networks. Paper presented at the IEEE Workshop on Mobile and Wireless Networks, Providence, RI.

[3] Caccamo, M., Zhang, Y., L., Sha, L., \& Buttazzo, G. (2002). An Implicit Prioitized Access Protocol for Wireless Sensor Networks. Proceedings of $23^{\text {rd }}$ IEEE Real-Time Systems Symposium, 39-48.

[4] Carzaniga, A., Rutherford, M., \& Wolf, A. (2004). A Routing Scheme for Content-Based Networking. Proceedings of IEEE 2004 International Conference on Computer Communications, $H K, 2,918-928$.

[5] Chai, Y., \& Ye, D. (2007). The Design and Implementation of a Scalable Wireless Video Streaming System Adopting TCP Transmission Mode. Proceedings of the 7th IEEE International Conference on Computer and Information Technology, Fukushima, 534-538.

[6] Chen, J., Chan, S., \& Li, V. (2004). Multipath routing for video delivery over bandwidth-limited networks. IEEE Journal on Selected Areas of Communications, 22(10), 1920-1932.

[7] Chen, M., Leung, V., Mao, S., \& Yuan, Y.,(2007). Directional Geographical Routing for RealTime Video Communications in Wireless Sensor Networks. Elsevier Computer Communications, 30(17), 3368-3383.

[8] Chu, M., Reich, J., \& Zhao, F. (2004). Distributed Attention in Large Scale Video Sensor Networks. Proceedings of IEEE Intelligent Distributed Surveilliance Systems, 61-65.

[9] Cosma, M., Pescaru, D., Ciubotaru, B., \& Todinca, D. (2006, May). Routing and Topology Extraction Protocol for a Wireless Sensor Network using Video Information. Paper presented at 3rd Romanian-Hungarian Joint Symposium on Applied Computational Intelligence, Timisoara, Romania.

[10] Fang, Q., Gao, J., \& Guibas, L. (2004). Locating and bypassing routing holes in sensor networks. Proceedings of the 23rd Conference of the IEEE Communications Society, China, 4, 2458-2468.

[11] Guo, S., Fan, C., and \& Little, T. (2008, July). Supporting Concurrent Task Deployment in Wireless Sensor Networks. Symposium on Network Computing and Applications(pp. 111-118). Los Alamitos, CA: IEEE Computer Society.

[12] Heinzelman, W., Chandrakasan, A., \& Balakrishnan, H. (2002). An application-specific protocol architecture for wireless microsensor networks. IEEE Transactions on Wireless Communication1(4), 660-670.

[13] Heinzelman, W., Chandrakasan, A., \& Balakrishnan, H. (2000). Energy-efficient Communication Protocol for Wireless Microsensor Networks. Proceedings of Hawaii International Conference System Sciences.

[14] Holman, R., Stanley, J., \& Ozkan-Haller, T. (2003). Applying Video Sensor Networks to Nearshore Enviroment Monitoring. IEEE Persave computing, 14-21.

[15] Intanagonwiwat, C., Govindan, R., \& Estrin, D. (2000, August). Directed diffusion: a scalable and robust communication paradigm for sensor networks. Paper presented at the $6^{\text {th }}$ Annual ACM/IEEE International Conference on Mobile Computing and Networking, Boston, MA. 
[16] Jia, W., Wang, T., Wang, G., \& Guo, M. (2007). Hole avoiding in advance routing in wireless sensor networks. Proceedings of the IEEE Wireless Communication \& Networking Conference, USA, 3519-3523.

[17] Kulkarni, P., Ganesan, D., Shenoy, P., \& Lu, Q. (2005). SensEye: A Multi-tier Camera Sensor Network. Proceedings of the $13^{\text {th }}$ annual ACM international conference on Multimedia, 229-238.

[18] Karp, B., \& Kung, H. (2000, August). GPSR: Greedy Perimeter Stateless Routing for Wireless Networks. Paper presented at the $6^{\text {th }}$ Annual International Conference on Mobile Computing and Networking, Boston, MA.

[19] Li, P., Gu, Y., \& Zhao, B. (2007, December). A Global-Energy-Balancing Real-time Routing in Wireless Sensor Networks. Paper presented at the 2nd IEEE Asia-Pacific Service Computing Conference, Tsukuba Science City, Japan.

[20] Li, S., Neelisetti, R., Liu, C., \& Lim, A. (2008, June). Delay-Constrained High Throughput Protocol for Multi-Path Transmission over Wireless Multimedia Sensor Networks. IEEE 2008 International Symposium on a World of Wireless, Mobile and Multimedia Networks (PP.1-8). Los Alamitos, CA: IEEE Computer Society.

[21] Little, T., Ishwar, P., \& Konrad, J. (2007). A Wireless Video Sensor Network for Autonomous Coastal Sensing. Proceedings of Conference on Coastal Environmental Sensing Networks.

[22] Little, T., Dib, P., Shah, K., Barraford, N., \& Gallagher, B. (2008). Using LED Lighting for Ubiquitous Indoor Wireless Networking. Proceedings of the $4^{\text {th }}$ IEEE Intl. Conf. on Wirless and Mobile Computing, Networking and Communications, Avignon, France

[23] Liu, Y., \& Das, S. (2006, November). Information-Intensive Wireless Sensor Networks: Potential and Challenges. IEEE Communications Magazine, 44(11), 142-147.

[24] Liu, X., Wang, Q., Sha, L., \& He, W. (2003). Optimal QoS Sampling Frequency Assignment for Real-Time Wireless Sensor Networks. Proceedings of 24th IEEE Real-Time Systems Symposium, 308-319.

[25] Navda, V., Kashyap, A., \& Ganguly, S. (2006). Real-time video stream aggregation in wireless mesh network. Proceedings of 17th International Symposium on Personal, Indoor and Mobile Radio Communications, Finland, 1-7

[26] Newsome, J., \& Song, D. (2003). GEM: Graph Embedding for Routing and Data-Centric Storage in Sensor Networks without Geographic Information. Proceedings of the First ACM Conf.: Embedded Networked Sensor Systems, USA, 76-88.

[27] Ould-Ahmed-Vall, E., Blough, D., Heck, B., \& Riley, G. (2005). Distributed global identification for sensor networks. Proceedings of $2^{\text {nd }}$ IEEE International Conference on Mobile Ad-hoc and Sensor Systems, Washington, DC.

[28] Pearlman, M., Haas, Z., Sholander, P., \& Tabrizi, S. (2000). On the Impact of Alternate Path Routing for Load Balancing in Mobile Ad Hoc Networks. Proceedings of the $1^{\text {st }} A C M$ International Symposium on Mobile Ad hoc Networking and Computing, Boston, MA, 3-10.

[29] Politis, I., Tsagkaropoulos, M., Dagiuklas, T., \& Kotsopoulos, S. (2007). Intelligent Packet Scheduling for Optimized Video Transmission over Wireless Networks. Proceedings ofthe 3rd International Mobile Multimedia Communications Conference, Nafpaktos, Greece, 329

[30] Politis, I., Tsagkaropoulos, M., Dagiuklas, T., \& Kotsopoulos, S. (2008). Power Efficient Video Multipath Transmission over Wireless Multimedia Sensor Networks. Mobile Networks and Applications, 13(3-4), 274-284.

[31] Rao, A., Ratnasamy, S., Papadimitriou, C., Shenker, S., \& Stoica, I. (2003). Geographic Routing without Location Information. Proceedings of the $9^{\text {th }}$ Annual International Conference on Mobile Computing and Networking, San Diego, CA. 
[32] Rowe, A., Goel, D., \& Rajkumar, R. (2007). FireFly Mosaic: A Vision-Enabled Wireless Sensor Networking System. Proceedings of the $28^{\text {th }}$ IEEE International Real-Time Systems Symposium, 459-468.

[33] Shin, J., Chin, M., \& Kim, C. (2006). Optimal Transmission Range for Topology Management Wireless Sensor Networks. Proceedings of International Conference on Information Networking, Japan, 3961, 177-185.

[34] Shu, L., Zhang, Y., Zhou, Z., Hauswirth, M., Yu, Z., \& Hyns, G. (2008). Transmitting and Gathering Streaming Data in Wireless Multimedia Sensor Networks within Expected Network Lifetime. Mobile Network Application, 13(3-4), 306-323.

[35] Sohrabi, K., Gao, J., Allawadhi, V., \& Pottie, G. (2000). Protocols for Self-organization of a Wireless Sensor Network. IEEE Personal Communications, 7(5), 16-27.

[36] Teixeira, T., Lymberopoulos, D., Culurciello, E., Aloimonos, Y., \& Savvides, A. (2006). A Lightweight Camera Sensor Network Operating on Symbolic Information. Proceedings of the first Workshop on Distributed Smart Cameras, Boulder, CO, USA.

[37] Teixeira, T., \& Savvides, A. (2007). Lightweight People Counting and Localizing in Indoor Spaces Using Camera Sensor Nodes. Proceedings of the first ACM/IEEE International Conference, 36-43.

[38] Wang, J., Masilela, M., \& Liu, J. (2007, December). Supporting Video Data in Wireless Sensor Networks. $9^{\text {th }}$ IEEE International Symposium on Multimedia (pp. 310-317). Los Alamitos, CA

[39] Wu, X., Cho, J., d'Auriol, B., \& Lee, S. (2007). Energy-aware routing for wireless sensor networks by AHP. Proceedings of IFIP Workshop on Software Technologies for Future Embedded \& Ubiquitous Systems, Greece, 446-455.

[40] Yu, F., Lee, E.,Choi, Y.,Park, S.,Lee, D., \& Tian, Y. (2007). A modeling for hole problem in wireless sensor networks. Proceedings of the International Wireless Communications and Mobile Computing Conference, USA, 370-375.

[41] Yu, Y., Govindan, R., \& Estrin, D. (2001, May). Geographical and energy aware routing: a recursive data dissemination protocol for wireless sensor networks. Unpublished UCLA Computer Science Department Technical Report UCLA/CSD-TR-01-0023, UCLA, CA.

[42] Zhang, L., Hauswirth, M., Shu, L., Zhou, Z., Reynolds, V., \& Han, G. (2008, June). Multi-priority Multi-Path Selection for Video Streaming in Wireless Multimedia Sensor Networks. Paper presented at the fifth International conference on Ubiquitous Intelligence and Computing, Oslo, Norway.

[43] Zhao, Y., Chen, Y., Li, B., \& Zhang, Q. (2007). Hop ID: A Virtual Coordinate-Based Routing for Sparse Mobile Ad Hoc Networks. IEEE Transactions on Mobile Computing, 6(9), 1075-1089. 